\title{
Brain MRI Segmentation using Canny Edge Detection Technique
}

\author{
Vandana Rajput ${ }^{1}$, Nirupma Tiwari ${ }^{2}$, Manoj Ramaiya ${ }^{3}$ \\ Student [M. Tech], SRCEM, Gwalior, India ${ }^{1}$ \\ Assistant Professor, Computer Science, SRCEM, Gwalior, India ${ }^{2}$ \\ H.O.D, Computer science, SRCEM, Gwalior, India ${ }^{3}$
}

\begin{abstract}
Image segmentation is a very tough technical process in the field of image processing. It is mandatory to go for the pre-processing before actual identification and segregation of ROI in MRI. Edge detection is based on the identification of the bounadries on the basis of similar brightness level and continuity of some pattern in the pixels. It is not possible to separate the some segment without applying the pre-processing of the image. . The applications of medical image segmentation are 3D reconstruction and quantitative analysis. Our experiment uses the MRI because this type of images give the most reliable image of the internal human organs like brain.. In this paper, the common problems of the image segmentation are explored and a novel method of image detection has been used.The classical image segmentation methods have been discussed for example gradient based operators were used usedfor edge detection, but these methods could deliver in the noisy surroundings. In the medical applications, the precision cannot be compromised with. To overcome these difficulties, we have proposed Canny edge detection technique prior to NonLocal Fuzzy C-Means Clustering technique for the segmentation. Quantifying brain structures in such large databases cannot be practically accomplished by expert neuroanatomists using hand-tracing. Rather, this research will depend upon automated methods that reliably and accurately segment and quantify large number of brain regions. At present, there is little guidance available to help clinical research groups in choosing such tools. This work is targeted to find out and establish more reliable segmentation technique as compared to expert hand tracing. The proposed approach consistently gives better results for various noise levels in the image compared to the reference schemes. This method is used for detecting brain region based on their energy function. In order to compare between them, one slice of MRI image tested with these methods. The traditional and proposed edge detection algorithms are implemented in MATLAB and results of proposed method are presented and compared with traditional approach. [1]
\end{abstract}

Keywords: Edge detection, Brain MRI images, Canny edge detector, manual tracing, neuroanatomists, Segmentation, Robust Fuzzy C-means clustering (RFCM), image segmentation, non-local, brain tissue.

\section{INTRODUCTION}

Image segmentation is one of the fundamental approaches of digital image processing. Image segmentation is the process of partitioning a digital image into multiple segments (sets of pixels also known as super-pixels). The goal of segmentation is to simplify and/or change the representation of an image into something that is more meaningful and easier to analyze. Image segmentation is typically used to locate objects and boundaries (lines, curves, etc.) in images. More precisely, image segmentation is the process of assigning a label to every pixel in an image such that pixels with the same label share certain characteristics. This part deals with the formation, acquisition and processing of images.

During past few years, brain tumor segmentation in magnetic resonance imaging (MRI) has become a popular research area in the field of medical imaging system. MRI is used in radiology for analyzing internal structures and makes easy to extract the required region. The method used in this paper is a hybrid approach i.e. combination of watershed method and edge detection method by using MATLAB as a tool to detect the tumor boundaries in MRI image for different cases of brain. The result of this method makes very clear for physician to distinguish the tumor portion for surgical planning. The efficiency and accuracy of the hybrid method is demonstrated by the experiments on the MRI brain images. Experimental results presented in this paper are obtained by using MATLAB.

\section{CANNY EDGEDETECTIONMETHOD}

Canny detector is optimal edge detector. This method can be put to use where the different stages are possible. The salient stages of the Canny Edge method are listed as under:

(a)The Canny edge detection starts with the application of Gaussian Filter.It smoothens the image.

(b)The second step is to calculate gradient magnitude

(c)The third step is marked by Non-maxima suppression

(d)The final step is following thresholding.[17]

The non- maxima removal includes, edge strength of current pixel is compared with pixel in +ve and -ve direction. If it is found that magnitude the pixel found to be larger than other pixels, the value of the magnitude is 
Vol. 4, Issue 2, February 2017

maintained otherwise the value is removed.The double threshold algorithm is used to identify the edges. If we use only low threshold, noisy maxima will get added and if we use high threshold, there is a possibility of true maxima might be missed. This the reason why double threshold is used in canny edge detection method. The strong edge points than high threshold are set as strong and points weaker than low threshold are suppressed and points between thresholds are set as weak.

In order to remove the noise, filtering the image is the step which cannot be ignored. Gaussian filter is convolved with image to obtain smooth image. Followed by the application sobel or prewitt operator to find gradient magnitude and orientation.Aftercalculating gradient magnitude we get blurred edges in the image. To convert the blurred edges into sharp edges we [17]

\section{IMPROVED NON-LOCAL FUZZY CLUSTERING SEGMENTATION ALGORITHM (NLFCM)}

Defining the neighbor of pixel $\mathrm{j}$ is a neighbor patch $\mathrm{Pj}$, then the similarity of pixel $i$ and $j$ is the gray-similarity of patch Piand $\mathrm{Pj}$, the weight value wijcan be calculated by the following equation:[1]

The distance between patch $\mathrm{Pi}$ and $\mathrm{Pjis}$ calculated as following:

$$
\left\|y\left(P_{i}\right)-y\left(P_{j}\right)\right\|_{2}^{2}=\sum_{p=1}^{\left|P_{i}\right|}\left(y^{(p)}\left(P_{i}\right)-y^{(P)}\left(P_{j}\right)\right)^{2}
$$

Among formula (8) and (9), Ziis and regularized constant, $\mathrm{h}$ is a smooth parameter, vector $\mathrm{y}(\mathrm{Pi})$ contain gray information of neighbor of pixel i, $y^{(\mathrm{p})}(\mathrm{Pi})$ is the $\mathrm{p}^{\text {th }}$ value of the vector.

Because non-local algorithm can get more space information of pixels without need for additional priori knowledge, we combined it with FCM algorithm, to get better ability to suppress noise. First, we assume that the pixels in the same tissue have similar neighborhood patch, we define the objective function with NL penalty term is as follows:[3]

$$
J_{R F C M}=\sum_{j \in \Omega} \sum_{k=1}^{C} u_{j k}^{q}\left\|y_{j}-v_{k}\right\|^{2}+\frac{\beta}{2} \sum_{j \in \Omega} \sum_{k=1}^{C} u_{j k}^{q} \sum_{n \in N_{j}^{R}} w_{j n} \sum_{l \in L_{k}} u_{n l}^{q}
$$

Compare with formula (6), we find that formula (1) can adjust the influence of neighborhood pixels around affecting on it automatically by a weight parameter Wij. That is to say, if the neighbor of pixel $\mathrm{i}$ and $\mathrm{n}$ is similar, they may belong to a same tissue, and the weight Wijwill increase. Otherwise, if two pixels are different, the influence of penalty term will reduce, i.e. pixel $n$ will have small effect on current pixel i.

Us Lagrange multiplier method, we can get the final membership function and cluster centers: [4]

$$
\begin{gathered}
\left.u_{j k}=\frac{\left(\left\|y_{j}-v_{k}\right\|^{2}+\beta \sum_{n \in N_{j}^{R}} w_{j n} \sum_{l \in L_{k}} u_{n l}^{q}\right)^{-1 /(q-1)}}{\sum_{i=1}^{C}\left(\left\|y_{j}-v_{k}\right\|^{2}+\beta \sum_{n \in N_{j}^{R}} w_{j n} \sum_{l \in L_{k}} u_{n l}^{q}\right)^{-1 /(q-1)}}\right) \\
v_{i}=\frac{\sum_{k=1}^{n}\left(u_{i k}\right)^{m} x_{k}}{\sum_{k=1}^{n}\left(u_{i k}\right)^{m}}
\end{gathered}
$$

The flow step of NLFCM is as follows:

Step1: Initial the clustering parameters: class number $\mathrm{C}$ cluster center $\mathrm{v}(\mathrm{t})$, and membership function $\mathrm{u}(\mathrm{t})$, set error value to break iteration $£>0$, iteration initial value $t=0$; Step2: Calculate the weight value wijby formula (8); Step3: Calculate the membership function $u(t+1)$ by formula (11);

Step4: Calculate the cluster center $v(t+1)$ by formula (12); Step5: Calculate the iteration condition, if $\|v(i+1)-v(i)\| \leq$ $£$, stop the iteration; Otherwise, $\mathrm{t}=\mathrm{t}+1$ and go to step2.

\section{PROPOSED APPROACH}

The proposed approach applies canny edge detection before applying the segmentation. The advantage of the proposed approach is we can get the better segmentation result for the noisy image. As the canny edge provides the better edges in noisy environments, edge information can be used in the non-local fuzzy c-means segmentation. [5] Defining the neighbor of pixel $\mathrm{j}$ is a neighbor patch $\mathrm{Pj}$, then the similarity of pixel $\mathrm{i}$ and $\mathrm{j}$ is the edge-similarity of patch Piand $\mathrm{Pj}$, the weight value wijcan be calculated by the following equation:

$$
w_{i j}=\frac{1}{Z_{i}} e^{-1 / h^{2}\left\|y\left(p_{i}\right)-y\left(p_{j}\right)\right\|_{2}^{2}}
$$

The distance between patch $\mathrm{Pi}$ and Pjis calculated as following:

$$
\left\|y\left(P_{i}\right)-y\left(P_{j}\right)\right\|_{2}^{2}=\sum_{p=1}^{\left|P_{i}\right|}\left(y^{(p)}\left(P_{i}\right)-y^{(P)}\left(P_{j}\right)\right)^{2}
$$

Among formula (13) and (14), Ziis and regularized constant, $h$ is a smooth parameter, vector $y(P i)$ contain edge information of neighbor of pixel $i, y^{(p)}(P i)$ is the $p^{\text {th }}$ value of the vector. 
Vol. 4, Issue 2, February 2017

Because non-local algorithm can get more space used was Windows 10.The standard NLFCM are tested information of pixels without need for additional priori and before this Canny Edge Detection Method is applied. knowledge, we combined it with FCM algorithm, to get The brain images are obtained from Brain webwebsite. better ability to suppress noise. First, we assume that the The Gaussian noise 5, 10,15,20,25,30 is added to the edges in the same tissue have similar neighborhood patch, original images. The results of this experiment are shown we define the objective function with NL penalty term is in fig.1. and fig.2.[16] as follows:

[1]

$$
J_{R F C M}=\sum_{j \in \Omega}^{C} \sum_{k=1}^{C} u_{j k}^{q}\left\|y_{j}-v_{k}\right\|^{2}+\frac{\beta}{2} \sum_{j \in \Omega} \sum_{k=1}^{C} u_{j k}^{q} \sum_{n \in N_{j}^{R}} w_{j n} \sum_{l \in L_{k}} u_{n l}^{q}
$$

Compare with formula (6), we find that formula (14) can adjust the influence of neighborhood pixels around affecting on it automatically by a weight parameter Wij. That is to say, if the neighbor of pixel edge $i$ and $n$ is similar, they may belong to a same tissue, and the weight Wijwill increase. Otherwise, if two edges are different, the influence of penalty term will reduce, i.e. edge pixel $n$ will have small effect on current pixel i.

Use Lagrange multiplier method, we can get the final membership function and cluster centers:[1]

$$
\begin{gathered}
u_{j k}=\frac{\left(\left\|y_{j}-v_{k}\right\|^{2}+\beta \sum_{n \in N_{j}^{R}} w_{j n} \sum_{l \in L_{k}} u_{n l}^{q}\right)^{-1 /(q-1)}}{\sum_{i=1}^{C}\left(\left\|y_{j}-v_{k}\right\|^{2}+\beta \sum_{n \in N_{j}^{R}} w_{j n} \sum_{l \in L_{k}} u_{n l}^{q}\right)^{-1 /(q-1)}} \\
\nu_{i}=\frac{\sum_{k=1}^{n}\left(\boldsymbol{u}_{i k}\right)^{m} x_{k}}{\sum_{k=1}^{n}\left(\boldsymbol{u}_{i k}\right)^{m}}
\end{gathered}
$$

The flow step of proposed approach is as follows:[1]

Step1: Initial the clustering parameters: class number $\mathrm{C}$ cluster center $v(t)$, and membership function $u(t)$, set error value to break iteration $£>0$, iteration initial value $\mathrm{t}=0$; Step2: Calculate the weight value wijby formula (13); Step3: Calculate the membership function $\mathrm{u}(\mathrm{t}+1)$ by formula (16);

Step4: Calculate the cluster center $v(t+1)$ by formula (17); Step5: Calculate the iteration condition, if $\|v(i+1)-v(i)\| \leq$ $€$, stop the iteration; Otherwise, $\mathrm{t}=\mathrm{t}+1$ and go to step2.

\section{EXPERIMENT RESEARCH AND ANALYSIS}

The experimental MATLAB codes were run on Intel core i3-50054 CPU, @2 GHz, 4GB Ram, the operating system

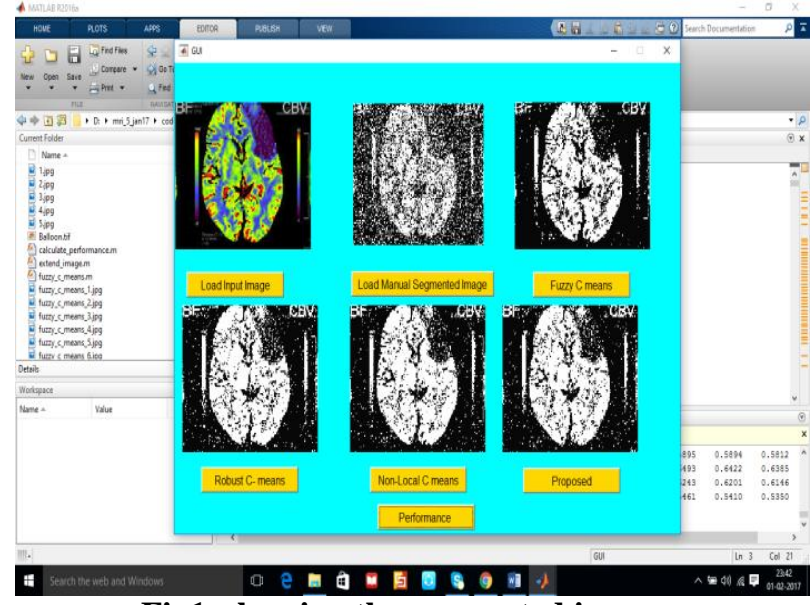

Fig1. showing the segmented images
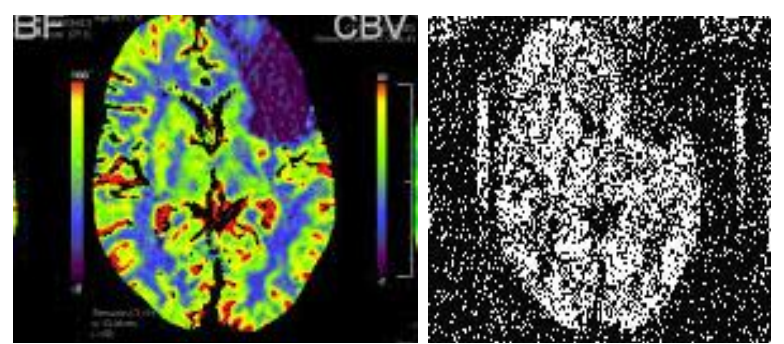

(A)ORIGINAL IMAGE

(B )IMAGE WITH NOISE
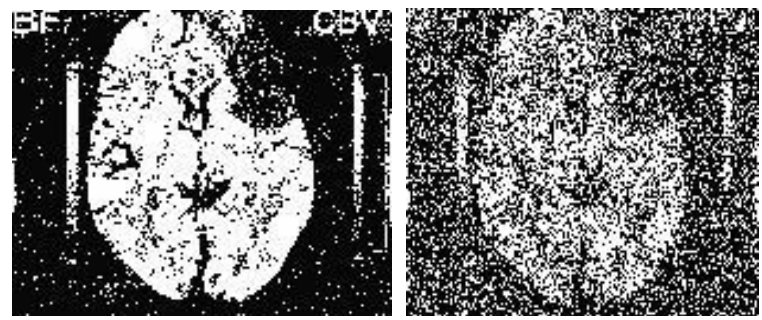

( C )FUZZY C-MEANS(D) ROBUST C-MEANS

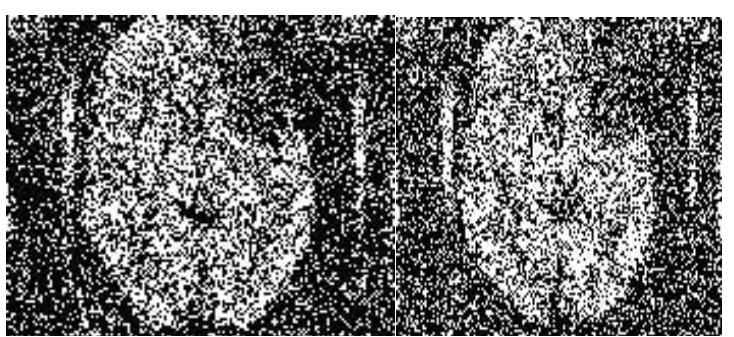

(E)NLFCM

(F)PROPOSED

FIG.2. COMPARISION OF THE RESULTS OF BASE ALGORITHMS AND PROPOSED ALOGORITHM 

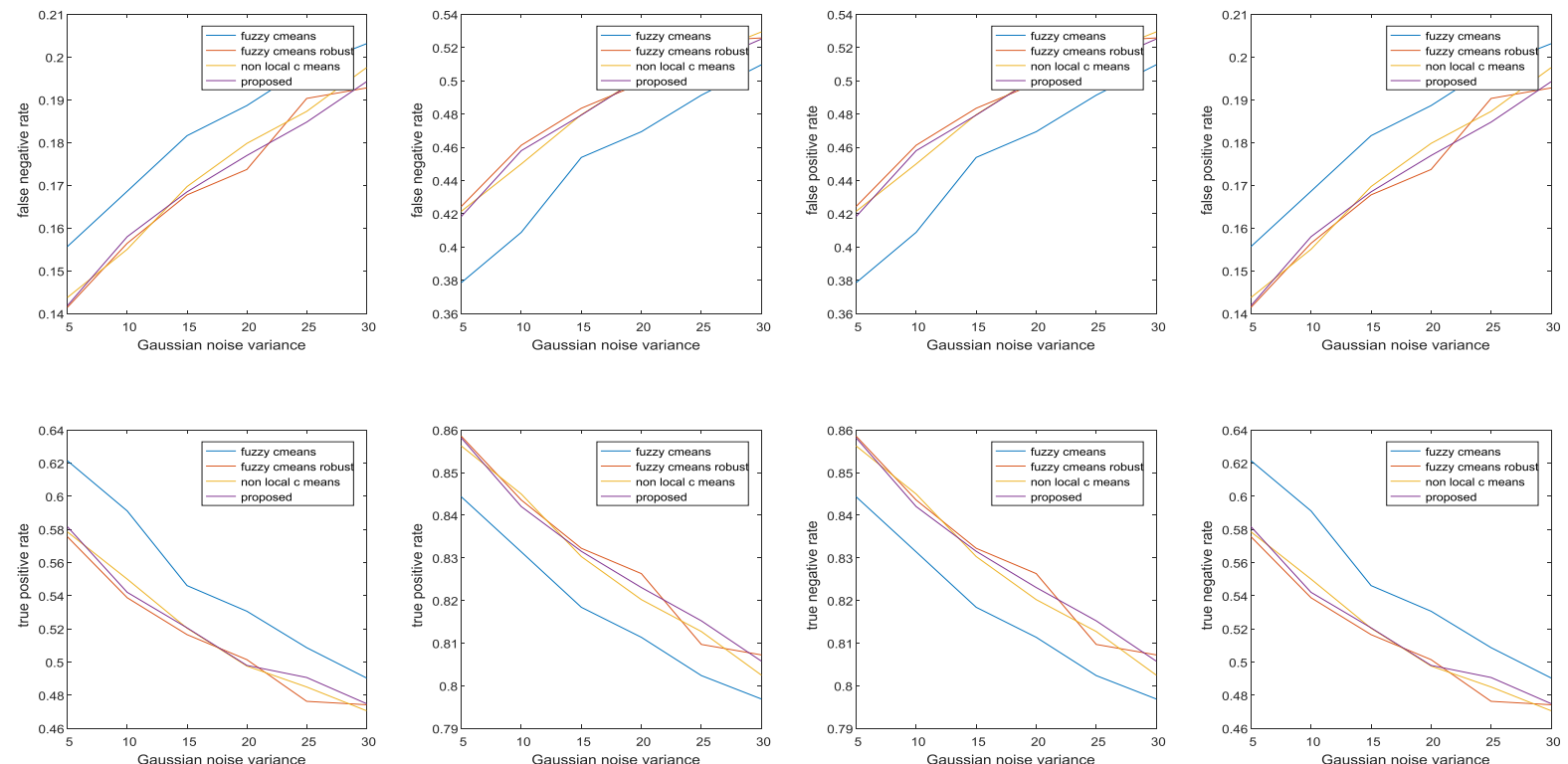

FIG.3. COMPARISON OF VARIOUS SEGMENTATION SCHEMES

TABLE 1. FALSE NEGATIVE RATE FALSE NEGATIVE RATE

\begin{tabular}{|l|l|l|l|l|l|l|}
\hline & Variance=5 & Variance=10 & Variance=15 & Variance=20 & Variance=25 & Variance=30 \\
\hline Fuzzy cmeans & 0.1557 & 0.1686 & 0.1816 & 0.1886 & 0.1976 & 0.2031 \\
\hline Fuzzy cmeans robust & 0.1415 & 0.1564 & 0.1678 & 0.1737 & 0.1903 & 0.1928 \\
\hline Non local cmeans & 0.1437 & 0.1550 & 0.1697 & 0.1798 & 0.1873 & 0.1975 \\
\hline Proposed & 0.1419 & 0.1580 & 0.1685 & 0.1770 & 0.1848 & 0.1942 \\
\hline
\end{tabular}

TABLE 2. FALSE POSITIVE RATE

\begin{tabular}{|l|l|l|l|l|l|l|}
\hline & Variance $=5$ & Variance $=10$ & Variance $=15$ & Variance $=20$ & Variance $=25$ & Variance $=30$ \\
\hline Fuzzy cmeans & 0.3786 & 0.4088 & 0.4539 & 0.4694 & 0.4914 & 0.5096 \\
\hline Fuzzy cmeans robust & 0.4244 & 0.4612 & 0.4835 & 0.4986 & 0.5237 & 0.5258 \\
\hline Non local cmeans & 0.4215 & 0.4500 & 0.4796 & 0.5025 & 0.5151 & 0.5294 \\
\hline Proposed & 0.4184 & 0.4579 & 0.4794 & 0.5020 & 0.5093 & 0.5251 \\
\hline
\end{tabular}

TABLE 3. TRUE POSITIVE RATE

\begin{tabular}{|l|l|l|l|l|l|l|}
\hline & Variance $=5$ & Variance $=10$ & Variance $=15$ & Variance $=20$ & Variance $=25$ & Variance $=30$ \\
\hline Fuzzy cmeans & 0.6214 & 0.5912 & 0.5461 & 0.5306 & 0.5086 & 0.4904 \\
\hline Fuzzy cmeans robust & 0.5756 & 0.5388 & 0.5165 & 0.5014 & 0.4763 & 0.4742 \\
\hline Non local cmeans & 0.5785 & 0.5500 & 0.5204 & 0.4975 & 0.4849 & 0.4706 \\
\hline Proposed & 0.5816 & 0.5421 & 0.5206 & 0.4980 & 0.4907 & 0.4749 \\
\hline
\end{tabular}

TABLE 4. TRUE NEGATIVE RATE

\begin{tabular}{|l|l|l|l|l|l|l|}
\hline & Variance $=5$ & Variance=10 & Variance=15 & Variance=20 & Variance=25 & Variance=30 \\
\hline F Fuzzy cmeans & 0.8443 & 0.8314 & 0.8184 & 0.8114 & 0.8024 & 0.7969 \\
\hline Fuzzy cmeans robust & 0.8585 & 0.8436 & 0.8322 & 0.8263 & 0.8097 & 0.8072 \\
\hline Non-local cmeans & 0.8563 & 0.8450 & 0.8303 & 0.8202 & 0.8127 & 0.8025 \\
\hline Proposed & 0.8581 & 0.8420 & 0.8315 & 0.8230 & 0.8152 & 0.8058 \\
\hline
\end{tabular}


Vol. 4, Issue 2, February 2017

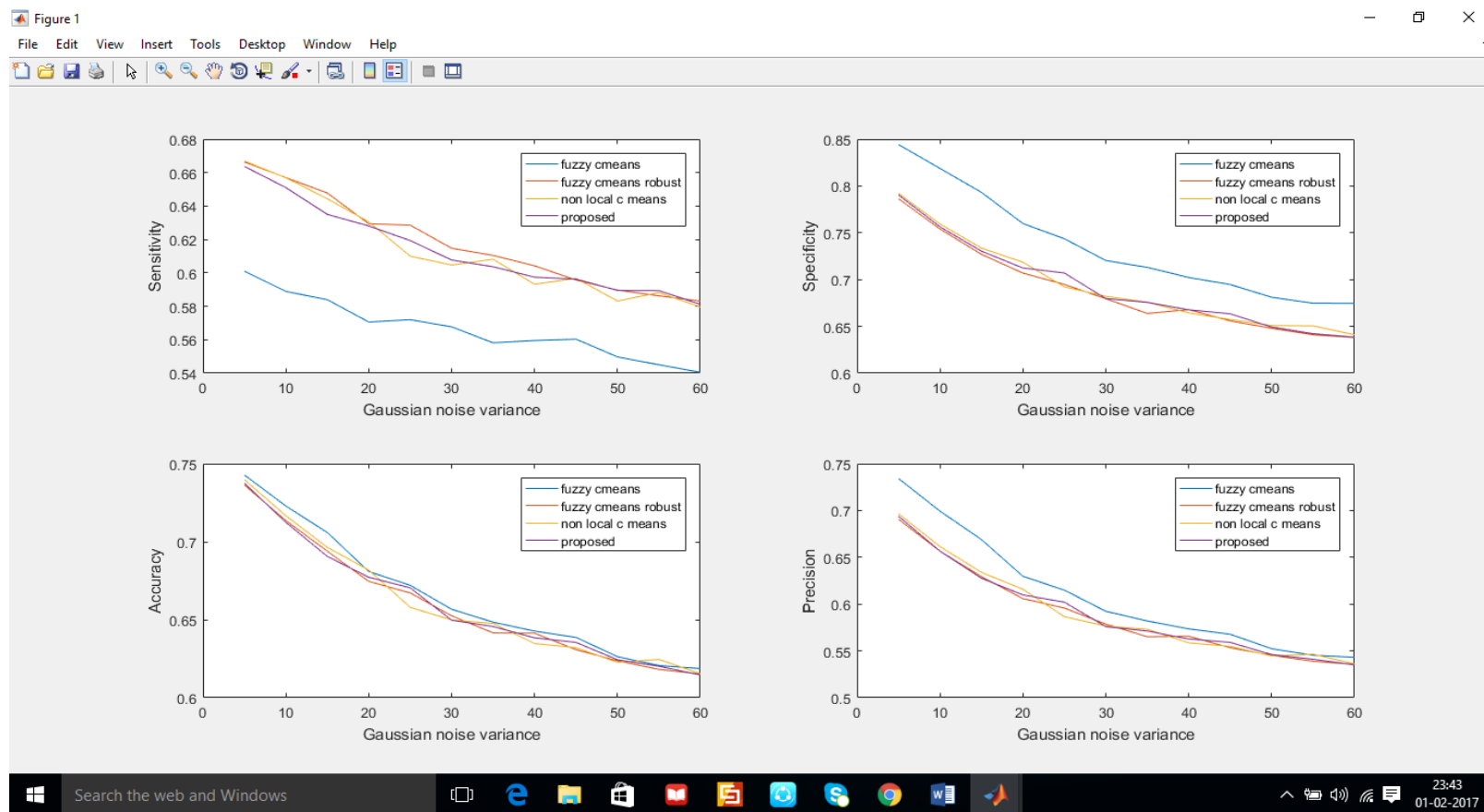

FIG.4. The Comparison Of Different Schemes Showing Sensitivity, Specificity, Accuracy And Precision

The graphs showing sensitivity, Specificity, Accuracy and Precision and can used in medical diagnosis. The presence of some signs of ailment suggest the the person may be sick. There are two possibilities the person may or may not have the disease. Physician and surgeons depend upon the diagnostic test to arrive at the conclusion about the diagnosis. Test result is either positive (diseased) or negative (healthy). False Positive: Healthy person incorrectly receives a positive (diseased) test result. False Negative: Diseased person incorrectly receives a negative (healthy) test result.[9]

\section{PERFORMANCE COMPARISON}

Fig. 1 shows the performance comparison of true positive rate (TPR), true negative rate (TNR), false positive rate (FPR), and false negative rate (FNR) between the various approaches (fuzzy c-means, fuzzy c-means robust, nonlocal c means and the proposed approach). The FPR is high for fuzzy c-means and low for the proposed approach at high Gaussian noises for the foreground which have the same pattern for false positive. FNR and FPR is high for the proposed approach for the background and the foreground. TPR for foreground and TNR for the background for the proposed approach and the fuzzy cmeans robust is nearly same whereas it is high for the fuzzy c-means. TPR for background and TNR for the foreground for the proposed approach is high and low for the fuzzy c-means. [15]

\section{CONCLUSION}

Segmentation is the one of the important problem in medical image processing to analyze the characteristics of the different regions. In this work, we implemented various approaches and proposed edge based non-local cmeans approach for segmentation. The proposed approach consistently gives better results for various noise levels in the image compared to the reference schemes.[1]

\section{REFERENCES}

[1] SUI Yuan, WEI Ying, IEEE ,MR Image Segmentation Algorithm Based On Non-Local Fuzzy C-Means Clustering, 978-1-47997016-2/15/\$31.00_c 2015 IEEE, 1117 - 1122.

[2] ChaoluFeng , Dazhe Zhao , Min Huang , 2016 Elsevier, Segmentation of longitudinal brain MR images using bias correction embedded fuzzy c-means with non-locally spatiotemporal Regularization, ..., C. Feng et al. / J. Vis. Commun. Image R. 38 (2016) 517-529, 517-529.

[3] ShaimaElnazer, Mohamed Morsy, MohyEldinA Abo-Elsoud IJSR, Brain Tumor Segmentation using hybrid of bothNetrosopic Modified Nonlocal Fuzzy C-mean and Modified Level sets, Volume 5 Issue 2, February 2016. Mr. Aditya P. Jadhav and Mr. Amar A. Dum, IRJET.

[4] Improved Fuzzy C-means Algorithm With Local Information And Trade-Off Weighted Fuzzy Factor for Image Segmentation,IRJET, $1047-1047,2015$.

[5] .Khalid A. Buragga, E. A. Zanaty, Sultan Aljahdali, ISCA, Fuzzy C-Means Algorithm Based on Gaussian Function for MagneticResonance Images (MRIs) Segmentation, 978-1-88084398-7 / copyright ISCA, CATA 2015 March 9-11, 2015, Honolulu, Hawaii, USA.

[6] YingdiGuo, Kunhong Liu, Qingqiang Wu, Qingqi Hong, Haiying Zhang, E-ISSN: 2224-2872, A New Spatial Fuzzy C-Means for Spatial Clustering, 369-381, Volume 14, 2015.

[7] WeilingCaiSongcanChen,Daoqiang Zhang, Corresponding author: Tel: +86-25-84896481-12106, Fax: +86-25-84498069. Email: s.chen@nuaa.edu.cn (S. C. Chen), Fast and Robust Fuzzy C-Means Clustering Algorithms Incorporating Local Information for Image Segmentation, 1-27.

[8] K.B.Vaishnavee and K.Amshakala, IEEE, An Automated MRI Brain Image Segmentation and Tumor Detection using SOM Clustering and Proximal Support Vector Machine Classifier, 2015 IEEE International Conference on Engineering and Technology (ICETECH), 20th March 2015, Coimbatore, TN, India. 
Vol. 4, Issue 2, February 2017

[9] Nupur J. Gandhi, Vandana J. Shah, Jr., RavindraKshirsagar, IEEE, Mean Shift Technique for Image Segmentation and Modified Canny Edge Detection Algorithm for Circle Detection, 246-250, International Conference on Communication and Signal Processing, April 3-5, 2014, India.

[10] M.Gomathi and Dr. P.Thangaraj, IJCSIS, A New Approach to Lung Image Segmentation using Fuzzy Possibilistic C-Means Algorithm, 222-228, (IJCSIS) International Journal of Computer Science and Information Security, Vol. 7, No. 3, March 2010.

[11] SaeedFazli and SaeedFathiGhiri, ISSN, A Novel Fuzzy C-Means Clustering with Hybrid Local and Non Local Spatial Information for Brain Magnetic Resonance Image Segmentation, 40-46, Journal of Applied Engineering (JOAE), 2 (4), April-2014 (Volume-II, Issue-IV).

[12] Muhammad AksamIftikhar, Abdul Jalil, SaimaRathore, MutawarraHussain, Wiley Periodicals, Inc., Robust Brain MRI Denoising and Segmentation Using Enhanced non-local Means Algorithm, 52-66, Vol. 24, 52-66 (2014).

[13] Hossam M. Moftah - Ahmad TaherAzar, springer, Adaptive kmeans clustering algorithm for MR breast image segmentation, 1917-1928, Neural Comput\&Applic (2014) 24:1917-1928.

[14] M. Ganesh and V. Palanisamy, ISSN, A Modified Adaptive Fuzzy C-Means Clustering Algorithm For Brain MR Image Segmentation, 1-8, International Journal of Engineering Research \& Technology (IJERT) Vol. 1 Issue 8, October - 2012 ISSN: 2278-0181.

[15] Irakykhalifa, AliaaYoussif, HowidaYoussry, International Journal of Computer Applications, MRI Brain Image Segmentation based on Wavelet and FCM Algorithm, 32-39, Volume 47- No.16, June 2012.

[16] XiaoqiangJi, Yang Li, JiezhangCheng,Yuanhua Yu, MeijiaoWang, IEEE, Cell Image Segmentation Based on an Improved Watershed Algorithm,433-437, 2015 8th International Congress on Image and Signal Processing (CISP 2015).

[17] NishthaParashar and NirupamaTiwari, International Journal of Signal Processing, Image Processing and Pattern Recognition, A Survey Of Digital Image Tampering Techniques, 91-96, Vol.8, No.10 (2015) 